\title{
Positive and Negative Roles for Soybean MPK6 in Regulating Defense Responses
}

\author{
Jian-Zhong Liu, ${ }^{1}$ Edward Braun, ${ }^{2}$ Wen-li Qiu, ${ }^{2}$ Ya-Fei Shi, ${ }^{1}$ Francismar C. Marcelino-Guimarães, ${ }^{3}$ \\ Duroy Navarre, ${ }^{4}$ John H. Hill, ${ }^{2}$ and Steven A. Whitham ${ }^{2}$ \\ ${ }^{1}$ College of Chemistry and Life Sciences, Zhejiang Normal University, Jinhua, China 321004; ${ }^{2}$ Department of Plant Pathology \\ and Microbiology, lowa State University, Ames 50011, U.S.A.; ${ }^{3}$ EMBRAPA Soja, Rodovia Carlos João Strass, Distrito de Warta, \\ CEP 86001-970, Londrina, PR, Brazil; ${ }^{4}$ Department of Plant Pathology, Washington State University, Prosser 99350, U.S.A.
}

Submitted 22 November 2013. Accepted 7 April 2014.

\begin{abstract}
It has been well established that MPK6 is a positive regulator of defense responses in model plants such as Arabidopsis and tobacco. However, the functional importance of soybean MPK6 in disease resistance has not been investigated. Here, we showed that silencing of GmMPK6 in soybean using virus-induced gene silencing mediated by Bean pod mottle virus (BPMV) caused stunted growth and spontaneous cell death on the leaves, a typical phenotype of activated defense responses. Consistent with this phenotype, expression of pathogenesis-related $(P R)$ genes and the conjugated form of salicylic acid were significantly increased in GmMPK6-silenced plants. As expected, GmMPK6-silenced plants were more resistant to downy mildew and Soybean mosaic virus compared with vector control plants, indicating a negative role of GmMPK6 in disease resistance. Interestingly, overexpression of GmMPK6, either transiently in Nicotiana benthamiana or stably in Arabidopsis, resulted in hypersensitive response (HR)-like cell death. The HR-like cell death was accompanied by increased $P R$ gene expression, suggesting that GmMPK6, like its counterpart in other plant species, also plays a positive role in cell death induction and defense response. Using bimolecular fluorescence complementation analysis, we determined that GmMKK4 might function upstream of GmMPK6 and GmMKK4 could interact with GmMPK6 independent of its phosphorylation status. Taken together, our results indicate that GmMPK6 functions as both repressor and activator in defense responses of soybean.
\end{abstract}

Mitogen-activated protein kinase (MAPK) cascades are highly conserved signaling modules across taxa. In plants, MAPK pathways are implicated in the regulation of growth and development, senescence, and programmed cell death, and in responses to a diversity of biotic and abiotic stresses (Colcombet and Hirt 2008; Ellis 2012; Innes 2001; Pitzschke

This is a journal paper of the Iowa Agriculture and Home Economics Experiment Station, Ames, IA, project number 3708.

Corresponding authors: S. A. Whitham; Telephone: +1.515.294.4952; Fax: +1.515.294.9420; E-mail: swhitham@iastate.edu; and J.-Z. Liu; Telephone: +1 86-0579-8228-8053; Fax: +1 6-0579-8228-2269; E-mail: jzliu@zjnu.cn

* The $\boldsymbol{e}$-Xtra logo stands for "electronic extra" and indicates that two supplementary figures are published online and that Figure 7 appears in color online.

(C) 2014 The American Phytopathological Society et al. 2009; Rasmussen et al. 2012). A MAPK signaling module consists of three protein kinases sequentially activated through phosphorylation by the upstream component: a MAP kinase kinase kinase (MAPKKK or MEKK), a MAP kinase kinase (MAPKK or MKK), and a MAP kinase (MPK) (Mészáros et al. 2006). MPK cascades act downstream of receptors to transduce extracellular stimuli into adaptive, intracellular responses (Pitzschke et al. 2009). Activation of an MPK can lead to changes in its subcellular localization and its interaction with and phosphorylation of transcription factors, thereby reprogramming gene expression (Hoang et al. 2012; Li et al. 2012a; Mao et al. 2011; Meng et al. 2013; Menke et al. 2004; Nguyen et al. 2012a and b; Pitzschke et al. 2009; Rasmussen et al. 2012).

Arabidopsis MPK3 and MPK6 are the most extensively studied MPK in plants. MPK3 and MPK6 are activated in response to a variety of environmental and developmental cues (Ahlfors et al. 2004; Asai et al. 2002; Bush and Krysan 2007; Cho et al. 2008; Dutilleul et al. 2012; Evrard et al. 2013; Galletti et al. 2011; Hord et al. 2008; Khan et al. 2013; Li et al. 2012a and b; Meng et al. 2012; Montillet et al. 2013; Takahashi et al. 2007; Teige et al. 2004; Wang et al. 2007, 2008; Wang et al. 2010; Xing et al. 2009; Xu and Chu 2012; Ye et al. 2013; Yoo et al. 2008; Zhou et al. 2009). Plant recognition of pathogens leads to rapid activation of MPK3 and MPK6. The flagellinderived peptide flg22 triggers a rapid and strong activation of MPK3 and MPK6 (Droillard et al. 2004). This activation occurs within $5 \mathrm{~min}$ of treatment, even in the presence of the translation inhibitor cycloheximide, indicating a direct link between receptors and the initiation of the MAPK signaling pathways (Asai et al. 2002). MPK6 is also activated by harpin proteins, which are encoded by hypersensitive response and pathogenicity genes in many plant-pathogenic bacteria (Desikan et al. 2001) and by chitin, a structural component of fungal cell walls (Wan et al. 2004). MPK6 activation is followed by the induction of pathogenesis-related $(P R)$ genes (Desikan et al. 2001). Silencing of MPK6 in Arabidopsis compromised resistance to an avirulent strain of Peronospora parasitica and avirulent and virulent strains of Pseudomonas syringae, suggesting that MPK6 plays roles in both effector-triggered immunity (ETI) and pathogen-associated molecular pattern-triggered immunity (PTI) (Menke et al. 2004).

Like their counterparts in Arabidopsis, salicylic acid (SA)induced protein kinase (SIPK, MPK6 ortholog) and woundinduced protein kinase (WIPK, MPK3 ortholog) are activated by various pathogens or pathogen-derived elicitors in tobacco (Desikan et al. 2001; Yang et al. 2001; Zhang et al. 2001). The gene-for-gene specific activation of SIPK and WIPK by 
Tobacco mosaic virus (TMV) and Avr9 suggests their roles in disease resistance (Zhang and Klessig 1998). NtMEK2 is an ortholog of Arabidopsis MKK4/MKK5 that functions upstream of SIPK and WIPK (Yang et al. 2001; Zhang et al. 2001). Expression of a constitutively active mutant of NtMEK2 $\left(\mathrm{NtMEK} 2^{\mathrm{DD}}\right.$ ) induces hypersensitive response (HR)-like cell death in tobacco, which is preceded by the activation of endogenous SIPK and WIPK. In addition, the NtMEK2-SIPK/WIPK cascade appears to control the expression of 3-hydroxy-3methylglutaryl CoA reductase $(H M G R)$ and L-phenylalanine ammonia lyase $(P A L)$. These two defense genes encode key enzymes in phytoalexin and SA biosynthesis pathways, indicating that a plant MAPK cascade controls multiple defense responses against pathogen invasion (Yang et al. 2001; Zhang et al. 2001). In support of this idea, silencing of the three known components in the NtMEK2-SIPK/WIPK pathway in tobacco attenuated $N$-gene-mediated TMV resistance (Jin et al. 2003). This observation indicated a positive role of the NtMEK2-SIPK/WIPK pathway in $N$-mediated resistance, possibly through regulating HR cell death.

Camalexin is an important phytoalexin whose production is critical for resistance against Botrytis cinerea in Arabidopsis, and MPK3 and MPK6 are required for its production. Conditional gain-of-function plants for Arabidopsis MKK4/MKK5 or MEKK1 and inducible, constitutive active forms of tobacco NtMEK2 (a homolog of MKK4/MKK5), NtMEK2 ${ }^{\mathrm{DD}}$ (phosphomimic), can activate MPK3/MPK6 and lead to camalexin accumulation in the absence of pathogen attack $(\mathrm{Xu}$ et al. 2008). The mpk3 and mpk6 mutants are compromised in the expression of genes required for camalexin biosynthesis and more susceptible to $B$. cinerea (Ren et al. 2008). These results indicate that the MPK3/MPK6 cascade regulates camalexin synthesis by stimulating transcription of the biosynthetic genes after pathogen infection (Ren et al. 2008).

MKK4 and MKK5 are not the only MKK that function upstream of MPK3/6 to stimulate camalexin biosynthesis. MKK9 is also implicated in camalexin production through activation of MPK3/6 (Xu et al. 2008). Camalexin accumulation and expression of genes required for camalexin production were severely compromised in $m k k 9$ seedlings. Additionally, constitutively active MKK9 exhibited elevated levels of camalexin and biosynthetic enzyme transcripts that were dependent on MPK3 and MPK6 (Xu et al. 2008). Conditional MKK9 gain-of-function plants have elevated MPK3 or MPK6 activity and produce higher levels of camalexin than MKK4 or MKK5 gain-offunction plants (Xu et al. 2008). The MKK9-MPK3/6 module is also involved in ethylene production and signaling (Bethke et al. 2009; Jos et al.2008; Liu and Zhang 2004; Yoo et al. 2008), salt stress responses, and senescence (Zhou et al. 2009), indicating that this MAPK signaling module regulates multiple biological responses. However, the MKKK that functions upstream of MKK9 remains to be identified. It has been established that the YODA-MKK4/5-MPK3/6 module regulates stomatal development and patterning (Bergmann et al. 2004; Wang et al. 2007).

BLAST searches revealed that there are at least $56 M P K, 80$ $M P K K$, and over $100 M P K K K$ in the soybean genome (Liu et al. 2011). Among these MAPK genes, only GmMPK4 has been functionally characterized (Liu et al. 2011). In this report, we combined virus-induced gene silencing (VIGS) with overexpression approaches to examine the roles of GmMPK6 in soybean defense responses. Silencing GmMPK6 resulted in spontaneous cell death and enhanced resistance against Soybean mosaic virus (SMV) and downy mildew (Peronospora manshurica). Interestingly, transient overexpression of GmMPK6 in Nicotiana benthamiana or stable overexpression in Arabidopsis caused HR-like cell death and activated defense responses. These results suggest that GmMPK6 is both an activator and repressor for defense responses. In addition, using bimolecular fluorescence complementation (BiFC), we showed that GmMKK4 interacted with GmMPK6 in planta and this interaction was independent of the activation state of GmMKK4, suggesting that, as in Arabidopsis, a GmMKK4GmMPK6 module exists in soybean.

\section{RESULTS}

Silencing GmMPK6 results in constitutive defense response.

MPK6 has been shown to play a positive role in disease resistance in other plant species such as Arabidopsis, tobacco, and tomato. To understand the role of GmMPK6 in disease resistance, GmMPK6 was silenced via Bean pod mottle virus (BPMV)-mediated VIGS (Zhang et al. 2010). There are two GmMPK6 homologues in soybean (Glyma02g15690 and Glyma07g32750) sharing 97\% overall identity. Therefore, both genes would be silenced simultaneously in our VIGS experiment. At 20 days postinoculation (dpi), a stunted stature and spontaneous cell death were observed on the systemic leaves of GmMPK6-silenced plants but not on the systemic leaves of empty-vector control plants (Fig. 1A to C). The silencing phenotypes were uniformly shown on all 10 silenced plants (five replicates, two plants per replicate). We repeated the silencing experiment three times and observed the same phenotypes. These phenotypes are similar to what has been observed on GmMPK4-silenced plants in which a constitutive defense response was activated (Liu et al. 2011). To confirm that these phenotypes resulted from GmMPK6 silencing, reverse-transcriptase quantitative polymerase chain reaction (RT-qPCR) was performed to demonstrate that GmMPK6 mRNA expression was, indeed, silenced (Fig. 1D). In addition, Western blot analysis was performed using an Arabidopsis MPK6 antibody to determine whether GmMPK6 was silenced at the protein level. GmMPK6 was clearly detected in vector control plants and plants silenced for GmMPK4 and GmMKK4 (Fig. 1F, lanes 1, 3, and 4) but it was almost undetectable in GmMPK6-silenced plants (Fig. $1 \mathrm{~F}$, lane 2), confirming further that GmMPK6 was, indeed, silenced.

To test whether defense responses were constitutively activated in GmMPK6-silenced plants, we quantified expression of GmPRI in vector control and GmMPK6-silenced plants by RT-qPCR. As expected, mRNA transcripts of GmPRl were constitutively induced in GmMPK6-silenced plants (Fig. 1E), suggesting that a defense response was activated in these plants. Constitutively activated defense responses are usually associated with increased production of SA (Chen and Klessig 1991). To test whether SA biosynthesis was induced when GmMPK6 was silenced, the levels of both SA and its bound form, SA glucoside (SAG), were determined in GmMPK6silenced and vector control plants. Interestingly, free SA levels were similar in $G m M P K 6$-silenced and vector control plants (Fig. 2A) but SAG levels increased 1.82 fold in GmMPK6silenced plants compared with vector control plants (Fig. 2B). Because free SA but not its SAG form activates defense responses, we could not determine whether the constitutively activated defense response is SA dependent.

\section{Silencing GmMPK6 enhances resistance against SMV and downy mildew.}

Constitutively activated defense responses usually enhance disease resistance (Wang et al. 2007). We previously showed that silencing $G m M P K 4$ constitutively activated defense responses, resulting in enhanced resistance to SMV and downy mildew (Liu et al. 2011). To test whether silencing GmMPK6 
similarly enhances resistance against soybean pathogens, GmMPK6-silenced and empty-vector control plants were inoculated with an infectious clone of SMV expressing the $\beta$ glucuronidase (GUS) reporter protein (SMV-N-GUS) via a biolistic approach (Wang et al. 2006). Three leaves from three independent vector control plants or GmMPK6-silenced plants (nine leaves in total) were inoculated with SMV-N-GUS. At 3 dpi, the SMV-N-GUS infection was visualized by GUS staining (Fig. 3A and B), and the diameters of GUS foci were measured (Fig. 3C). The sizes of GUS foci were significantly decreased (Student's $t$ test, $P<0.01$ ) by 3.6-fold in GmMPK6silenced leaves compared with vector control leaves, demonstrating that silencing GmMPK6 enhanced resistance against SMV infection. As a control, 35-GUS was expressed equally well in GmMPK6-silenced and vector control leaves using biolistics (Supplementary Fig. S1). This control indicates that the reduced size of SMV-N-GUS foci on the leaves of GmMPK6-silenced plants was, indeed, due to activated defense responses and not to changes that affected transient expression driven by the $35 \mathrm{~S}$ promoter.

We then tested the effect of GmMPK6 silencing on resistance to downy mildew infection. In all, 10 vector control plants and 10 GmMPK6-silenced plants at 21 dpi were used in this experiment. Chlorotic lesions caused by $P$. manschurica infection were observed on leaves of vector control plants at 7 dpi (Fig. 4A). However, no lesions were observed on the leaves of GmMPK6-silenced plants (Fig. 4B). To further confirm these results, $P$. manschurica mycelia were observed within infected leaves using a $\mathrm{KOH}$-aniline blue staining procedure and fluorescence microscopy. Abundant hyphae were present in mesophyll cells of vector control plants with chlorotic lesions (Fig. 4C), whereas no hyphae were observed within the mesophyll of the GmMPK6-silenced plants (Fig. 4D). Together, these results clearly indicated that the GmMPK6 silencing enhanced resistance to two unrelated obligate pathogens, SMV and P. manshurica.

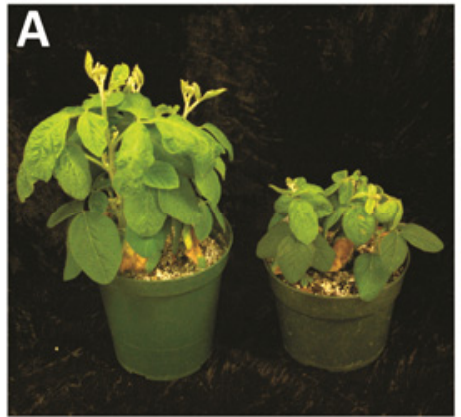

D

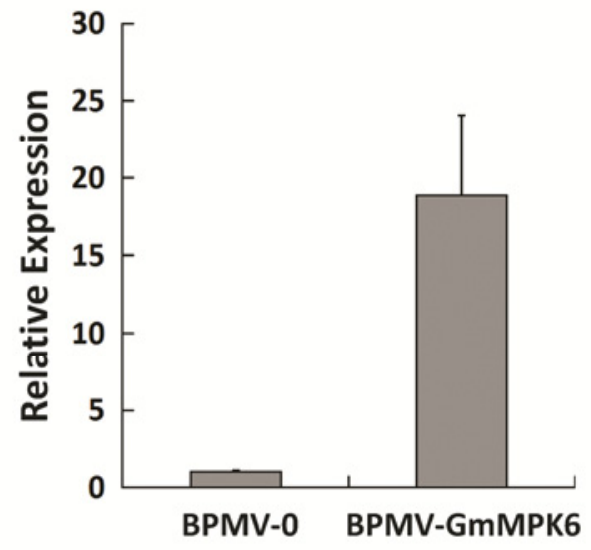

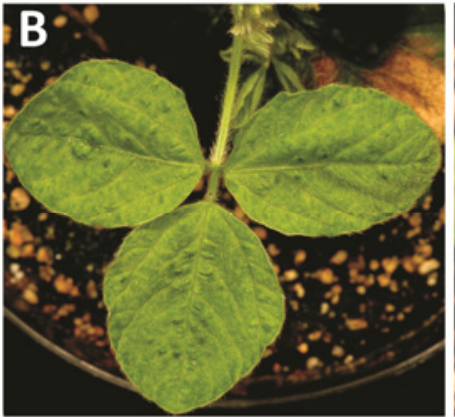

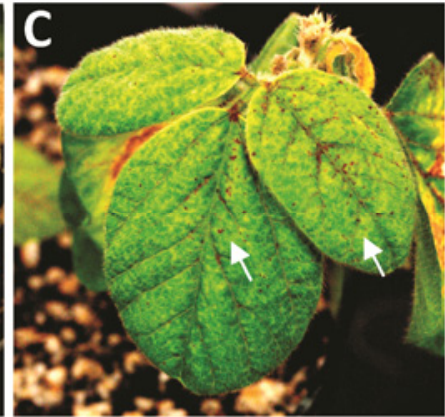

E

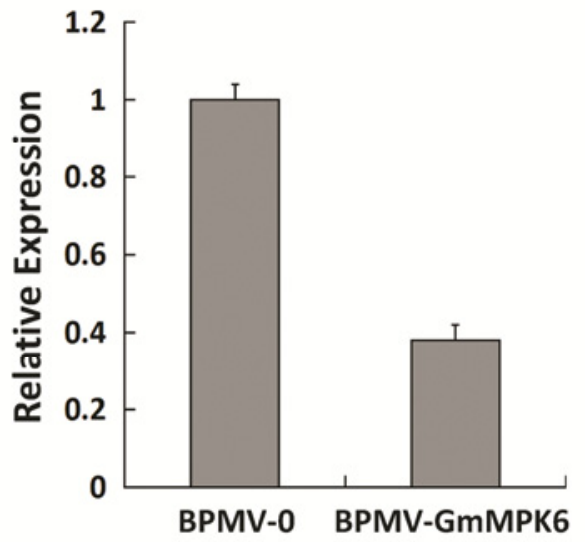

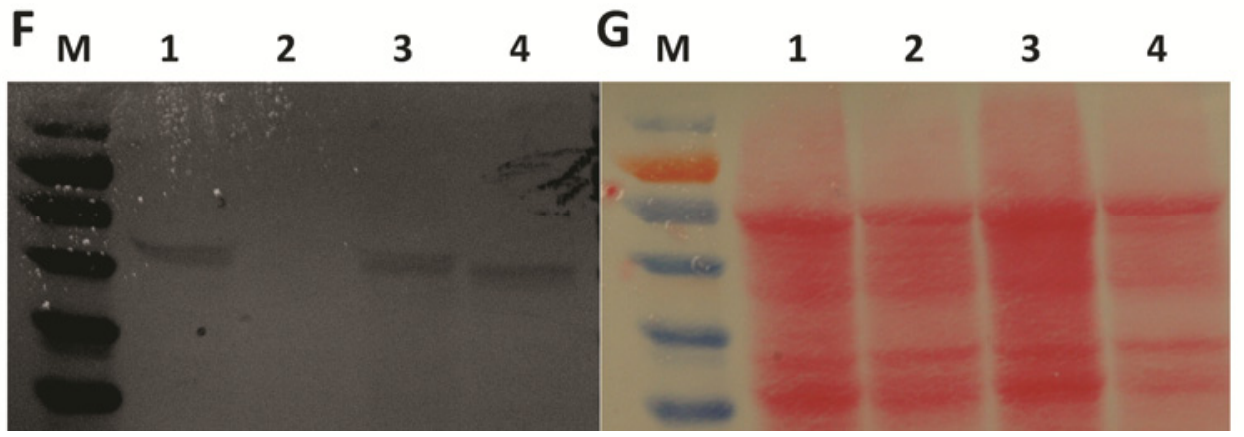

Fig. 1. Silencing GmMPK6 results in stunted stature and cell death in soybean. A, Stunted stature; B, empty Bean pod mottle virus (BPMV) vector control plant without cell death on the leaves; $\mathbf{C}$, cell death on the leaves of a GmMPK6-silenced plant; D, reverse-transcriptase quantitative polymerase chain reaction showing that transcript level of GmMPK6 was reduced in GmMPK6-silenced plants compared with vector control plant. The $\beta$-actin gene was used as the endogenous reference. E, $P R 1$ was induced in GmMPK6-silenced plants. F, Western blot analysis of GmMPK6 protein accumulation in empty vector control (BPMV-0) plants (lane 1) and GmMPK6-silenced plants (lane 2). GmMPK4-and GmMKK4-silenced plants served as controls (lanes 3 and 4). G, Ponceau $\mathrm{S}$ was used to stain the membrane used for Western blot analysis as a loading and transfer control. 
A

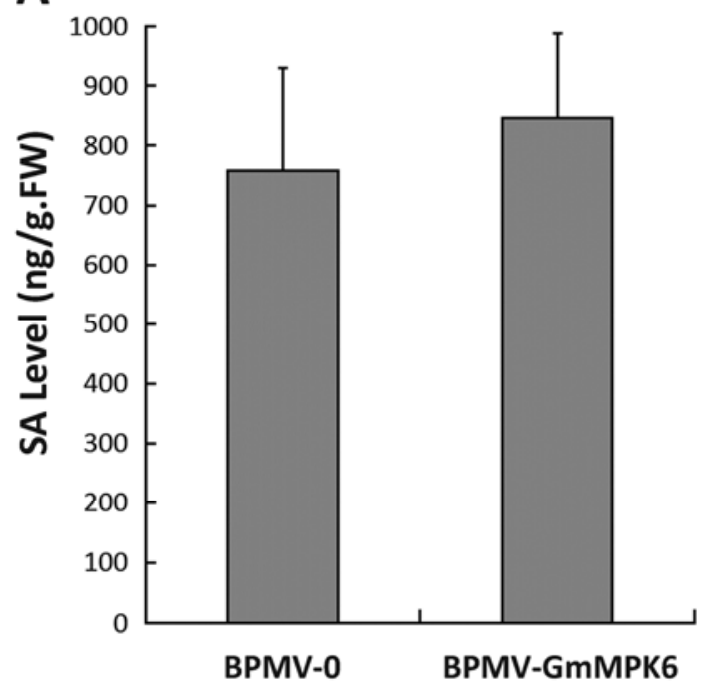

B

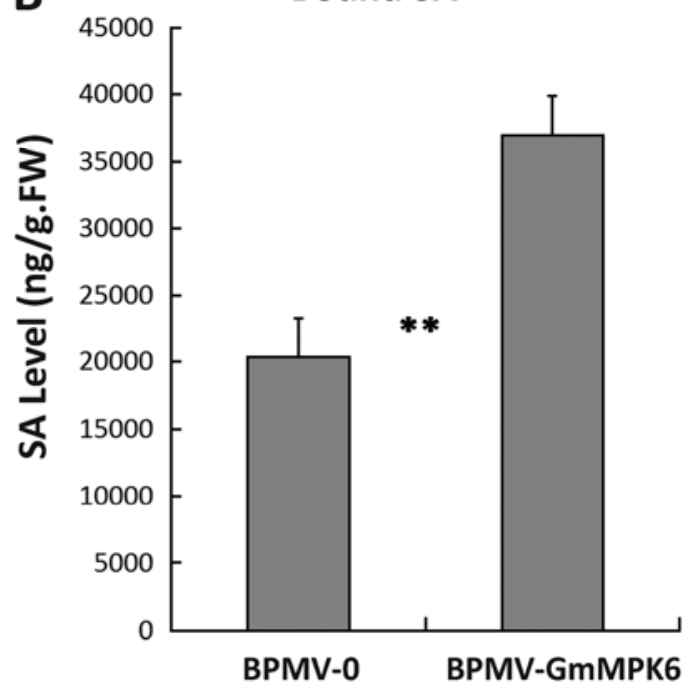

Fig. 2. Overaccumulation of bound salicylic acid (SA) but not free SA in GmMPK6-silenced soybean plants. Both A, free SA and B, bound SA glucoside levels were quantified in GmMPK6-silenced and vector control plants at 20 days postinoculation with Bean pod mottle virus (BPMV). Error bars represent standard deviation for three independent samples; ** indicates $P<0.01$, Student's $t$ test; FW $=$ fresh weight.
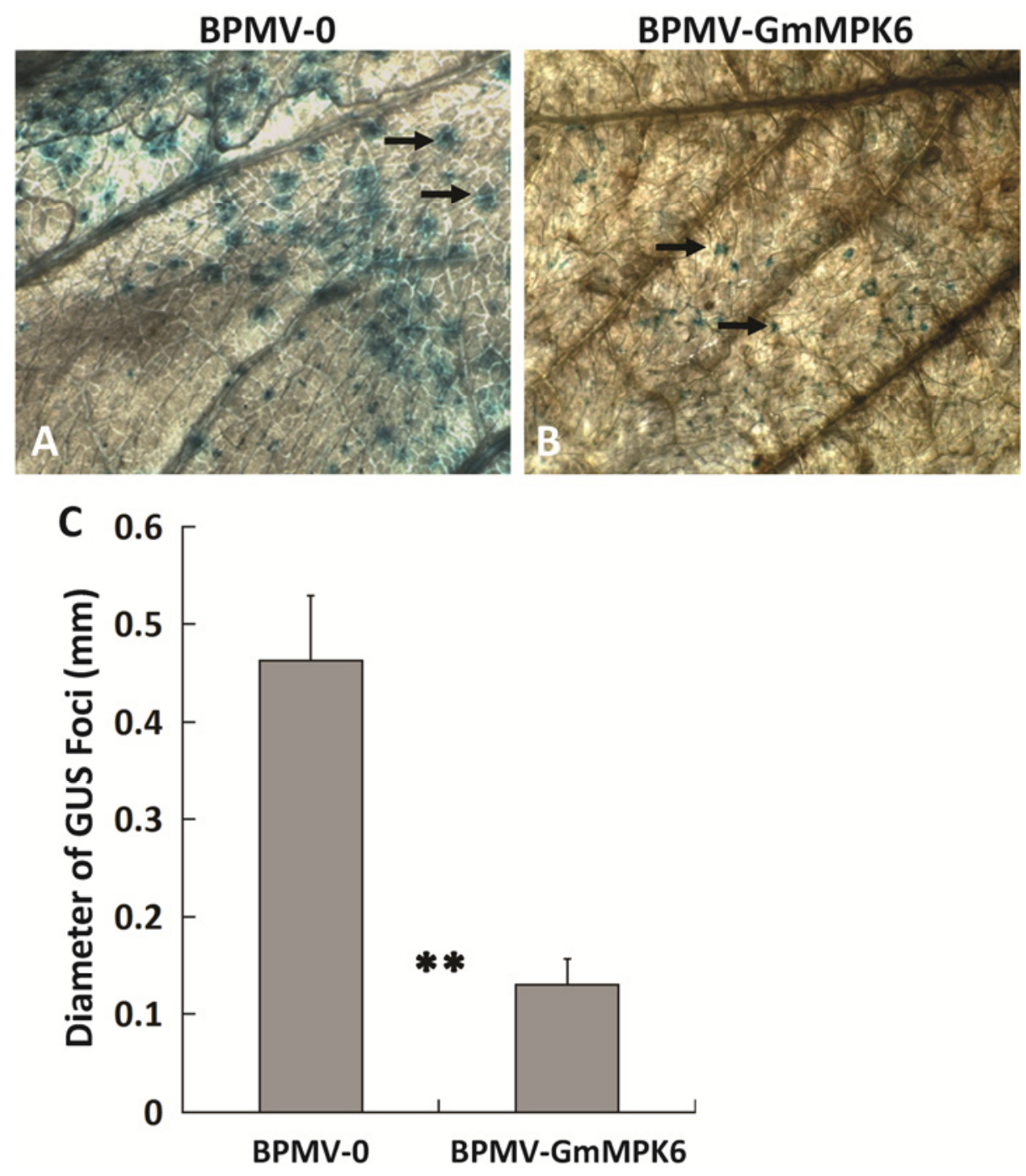

Fig. 3. Silencing GmMPK6 enhances the resistance of soybean plants against Soybean mosaic virus (SMV). At 18 day postinoculation (dpi) with Bean pod mottle virus (BPMV), SMV-N- $\beta$-glucuronidase (GUS) was biolistically delivered into detached leaves of either A, BPMV-0 or B, BPMV-GmMPK6 plants. C, Diameters of GUS foci on GmMPK6-silenced plants were significantly smaller than on vector control plants. Images were taken at 3 dpi with SMV-NGUS. Arrows point to the GUS foci and ** indicate significance at $P<0.01$ by Student's $t$ test. 
Transient overexpression of GmMPK6 in $N$. benthamiana or stable overexpression of GmMPK6 in Arabidopsis results in HR.

It has been reported that MPK6 functions as a positive regulator of cell death and disease resistance (Colcombet and Hirt 2008; Pitzschke et al. 2009; Rasmussen et al. 2012). However, our VIGS results suggested that GmMPK6 is a negative regulator of soybean defense responses. To test whether GmMPK6 has a positive role in cell death, we transiently overexpressed GmMPK6 in N. benthamiana leaves. Overexpressing GmMPK6 caused HR-like cell death on $N$. benthamiana leaves (Fig. 5), suggesting that GmMPK6 functions as both positive and negative regulator of cell death and defense response.

To further investigate this idea, transgenic Arabidopsis plants overexpressing GmMPK6 driven by the $35 \mathrm{~S}$ promoter were generated. Interestingly, six of 15 transgenic plants showed severe cell death phenotypes on their leaves (Fig. 6A to C). These plants were smaller in stature compared with wild-type (WT) plants and eventually died before bolting. RT-PCR results showed that $G m M P K 6$ was highly expressed and $P R$ genes were highly induced in these transgenic Arabidopsis plants (Fig. 6; Supplementary Fig. S2), indicating a positive role of GmMPK6 in cell death and defense responses.

To examine the effects of GmMPK6 overexpression on the expression of endogenous AtMPK6, RT-PCR was performed using the RNAs extracted from both WT and transgenic plants. The AtMPK6 was expressed higher in GmMPK6-overexpressing plants than in WT Col-0, indicating that overexpressing GmMPK6 induced the expression of AtMPK6.

\section{Overexpressing $G m M K K 4 / 5$ also induces HR on the leaves of $N$. benthamiana.}

NbMEK 2 or AtMKK4/5 functions upstream of NbMPK6 or AtMPK6, respectively, and overexpressing either $N b M E K 2$ or AtMKK4 results in cell death (Yang et al. 2001). We were interested to know whether GmMKK4 has functions similar to its homologs in Arabidopsis and N. benthamiana. To investigate this, the AtMKK4 amino acid sequence was used in a BLAST search to identify its closest homolog in soybean, which we refer to as GmMKK4 (Glyma07g00520). GmMKK4 was transiently overexpressed $N$. benthamiana leaves. Like its homologs from other plant species, overexpressing $G m M K K 4$ resulted in HR in the infiltrated area, which was not seen when the 35S-GFP control construct was infiltrated (Fig. 5).

The serine/threonine phosphorylation sites are highly conserved among MKK4s from different species. Replacement of the serine and threonine phosphorylation sites of AtMKK4 or NtMEK2 with negatively charged aspartic acid (MKK4 ${ }^{\mathrm{DD}}$ or MEK2 ${ }^{\mathrm{DD}}$ ) constitutively activates these kinases (Yang et al. 2001). In contrast, replacing the serine and threonine with
BPMV-0

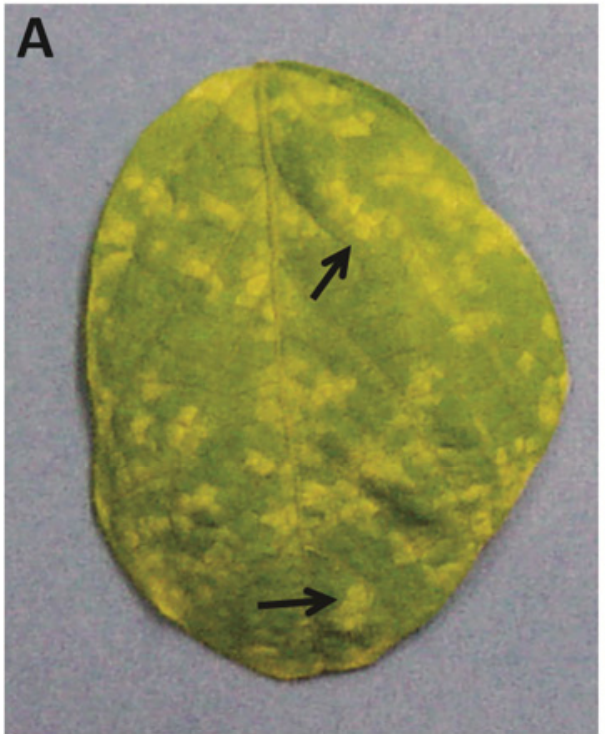

BPMV-0

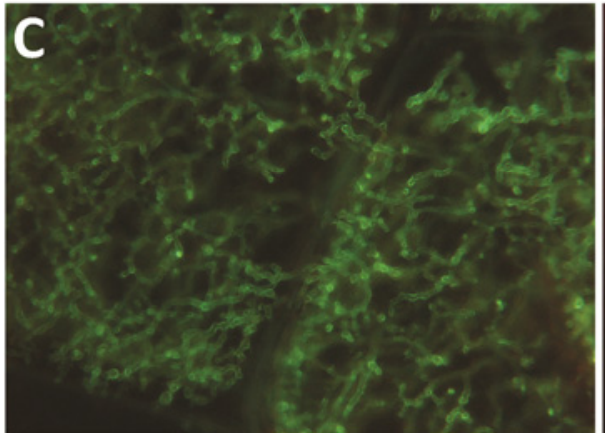

BPMV-GmMPK6

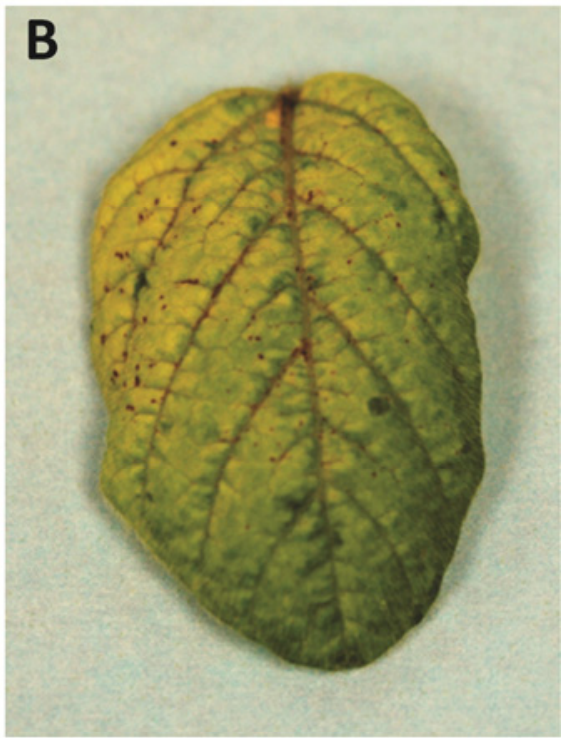

BPMV-GmMPK6

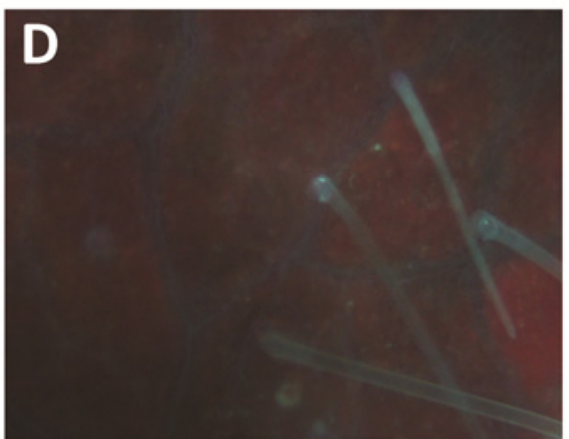

Fig. 4. Silencing GmMPK6 enhances the resistance of soybean plants against downy mildew. Chlorotic lesions typical of soybean downy mildew (arrows) were detected on A, the leaves of Bean pod mottle virus (BPMV)-0 vector control plants but not on B, the leaves of GmMPK6-silenced plants at 1 week after inoculation with Peronospora manschurica. P. manschurica hyphae were observed in $\mathbf{C}$, the mesophyll of BPMV-0 vector control plants but not in D, the mesophyll of GmMPK6-silenced plants at 1 week after inoculation with $P$. manschurica. 
positively charged lysine and arginine (MKK4 ${ }^{\mathrm{KR}}$ and MEK2 ${ }^{\mathrm{KR}}$ ) abolishes phosphorylation, cell death, and defense responses (Yang et al. 2001). To test whether this is also true for GmMKK4, we generated GmMKK4 ${ }^{\mathrm{DD}}$ and $\mathrm{GmMKK} 4^{\mathrm{KR}}$ and tested their ability to induce cell death (Fig. 5). GmMKK4 ${ }^{\mathrm{DD}}$ induced a faster HR that was observed at 2 dpi compared with 3 to 4 dpi for GmMPK6 or GmMKK4. In contrast, GmMKK $4{ }^{\mathrm{KR}}$ could not induce cell death on $N$. benthamiana leaves. These results indicated that the soybean MKK4-MPK6 signaling module has functions similar to its counterparts in Arabidopsis, tomato, and N. benthamiana.

\section{GmMKK4 interacts with GmMPK6 in vivo.}

The results shown above raised a possibility that an MKK4MPK6 module might function in soybean defense. However, these data do not provide evidence that GmMKK4 and GmMPK6 interact with each other. To determine whether they interact, $\mathrm{BiFC}$ was performed by co-bombarding onion epidermal cells with reciprocal combinations of the amino-terminal half of yellow fluorescence protein (nYFP) and c-terminal half of YFP (cYFP) fusions to either GmMKK4 or GmMPK6. Regardless of cYFP or nYFP fusion combination, YFP signals were detected in both the cytosol and the nucleus when GmMKK4 was co-expressed with GmMPK6 (Fig. 7, top row). As a positive control, a strong YFP signal was observed in the nucleus when nYFP-AtbZIP and cYFP-AtbZIP were co-expressed (data not shown) (Liu et al. 2011). As expected, no YFP signal was detectable when nYFP-GmMPK6 was co-expressed with cYFP-GmMKK6 (Glyma10g15850) (Fig. 7, bottom row). Together, these results indicated that GmMKK4 might function upstream of GmMPK6 to regulate defense response in soybean.

To test whether the mutations of GmMKK4 phosphorylation sites have an effect on GmMKK4 and GmMPK6 interaction, we performed BiFC assays between GmMPK6 and GmMKK4 $4^{\mathrm{DD}}$ or $\mathrm{GmMKK} 4^{\mathrm{KR}}$. Regardless of phosphorylation status, GmMPK6 interacted with both GmMKK4 ${ }^{\mathrm{DD}}$ (Fig. 7, second row) and GmMKK $4{ }^{\mathrm{KR}}$ (Fig. 7, third row), demonstrating that mutations on phosphorylation sites do not interfere with interaction of GmMKK4 with GmMPK6.

\section{DISCUSSION}

\section{Functional difference between GmMPK6}

and MPK6s in other plant species.

It has been well established that MPK6 in plants is a positive regulator of disease resistance (Colcombet and Hirt 2008; Pitzschke et al. 2009; Rasmussen et al. 2012). Our results showed that GmMPK6 functions as both positive and negative regulator in cell death and disease resistance. However, it is not unexpected that an MPK in plants can play opposing roles in regulating defense responses. For example, OsMPK6, an AtMPK4 homolog in rice, functions as both an activator and a repressor in rice resistance against Xanthomonas oryzae pv. oryzae (Shen et al. 2010). Either overexpression or knockout of OsMPK6 resulted in the activation of SA-dependent resistance against bacterial pathogens, indicating that OsMPK6 is a Janus-faced player in disease resistance (Shen et al. 2010). These results suggest that the MPK are major signaling components immediately downstream of immune receptors. The expression or activity of these MPK must be tightly controlled to ensure the activation of defenses in the presence of pathogens and to prevent the inappropriate activation of defenses in the absence of pathogens, which would occur at the expense of normal growth and development (Liu et al. 2011).

In Arabidopsis, MPK3 or MPK6 (positive regulators of defense) and MPK4 (negative regulator of defense) are simulta- neously activated by various stimuli (Pitzschke et al. 2009). This illustrates the complex network that controls plant defenses, in which both positive and negative regulators are involved, and the activation of defense responses is not necessarily correlated with only the activation of positive regulators. The activation of negative regulators, such as MPK4, could dampen or prevent overactivation of defense responses in order to maintain a proper level of defense responses. The alternative explanation is that MPK4 in Arabidopsis could also serve a positive role in disease resistance.

Menke and associates (2004) reported that silencing AtMPK6 in Arabidopsis compromised resistance to an avirulent strain of $P$. parasitica and avirulent and virulent strains of Pseudomonas syringae, suggesting that AtMPK6 functions in both ETI and PTI. Although AtMPK6-silenced plants exhibited enhanced disease susceptibility, their ability to develop systemic acquired resistance or induced systemic resistance was unaffected (Menke et al. 2004). Jin and associates (2003) reported that suppression of all three known components in the NtMEK2-SIPK/WIPK pathway attenuated $N$-gene-mediated TMV resistance. In addition, it has been shown that MPK6 contributes to nonhost resistance in Arabidopsis and rice (Okawa and Ishikawa 2013). Interestingly, a negative role of MPK6 in basal resistance has been reported recently for tobacco SIPK, a homolog of MPK6 (Kobayashi et al. 2010). Silencing WIPK and SIPK in tobacco enhances basal resistance against TMV but breaks $N$-mediated resistance (Kobayashi et al. 2010). Consistent with this observation, our results showed that knocking down GmMPK6 resulted in enhanced basal resistance against two unrelated pathogens in soybean (Figs. 3

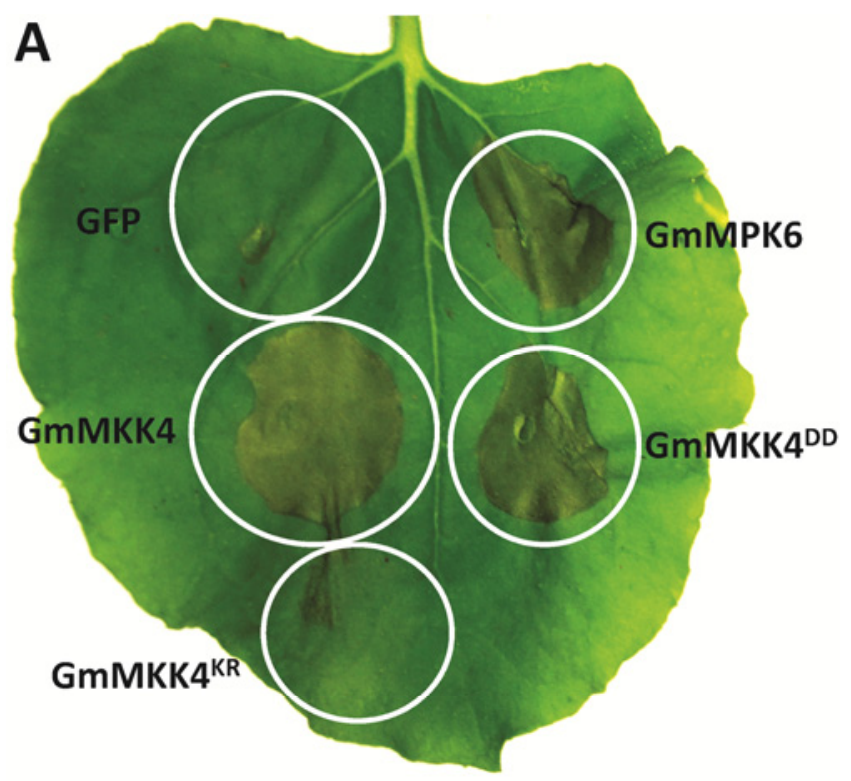

\section{B GmMKK4 GmMKKDD GmMKK4 ${ }^{\mathrm{KR}}$

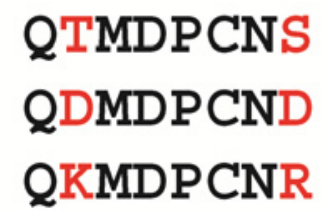

Fig. 5. The GmMKK4-GmMPK6 module positively regulates hypersensitive response (HR)-like cell death in Nicotiana benthamiana. A, Transient overexpression of GmMPK6 and GmMKK4 or its constitutively activated form, GmMKK4 ${ }^{\mathrm{DD}}$ but not its inactive form, $\mathrm{GmMKK} 4^{\mathrm{KR}}$, resulted in HR-like cell death. The unfused green fluorescent protein construct was used as a negative control. The leaf was photographed at 3 days post-infiltration. B, Amino acid sequences showing the phosphorylation sites of GmMKK4 (red) and its mutated versions. 
and 4). However, Rsv1-mediated resistance against SMV was not affected in GmMPK6-silenced plants (data not shown), raising the possibility that MPK6 homologs may function differently in different plant species.

The free SA level may account for the difference of phenotypes in severity between MPK4- and MPK6-silenced plants.

We previously showed that silencing GmMPK4 activated SA-dependent defense responses, and both free SA and SAG were greatly induced in GmMPK4-silenced plants (Liu et al. 2011). Unlike in GmMPK4-silenced plants, only SAG but not free SA was induced in GmMPK6-silenced plants (Fig. 2), suggesting that the conversion of SAG to SA could be compromised in GmMPK6-silenced plants. However, the GmPRI gene was induced 18-fold in GmMPK6-silenced plants relative to vector control plants (Fig. 1E). It is unclear at the moment whether the GmPRl induction in GmMPK6-silenced plants was due to increased levels of SAG or it was induced in an SA-independent manner, given the fact that free SA was not induced in GmMPK6-silenced plants (Fig. 2). Even though silencing both GmMPK4 and GmMPK6 resulted in necrosis or cell death on the leaves of silenced plants, the symptoms were more severe in GmMPK4-silenced plants than in GmMPK6silenced plants (data not shown). The less severe symptoms of GmMPK6-silenced plants could be due to lower induction of SA and SAG (Fig. 2) (Liu et al. 2011). If this is true, GmMPK6 might be a weaker repressor of SA-dependent defense response than GmMPK4.

Germ tubes with multiple appressoria were observed on the leaf surface of downy mildew-infected GmMPK4-silenced plants (Liu et al. 2011). This result indicated that downy mildew could not penetrate the cell wall of GmMPK4-silenced plants and the altered cell wall structures or components are at least partly, if not fully, responsible for the resistance against downy mildew. However, germ tubes that produced multiple appressoria were not observed on GmMPK6-silenced plants, indicating that a mechanistic difference might explain the resistance against downy mildew between GmMPK4- versus GmMPK6-silenced plants.

\section{Is enhanced expression of $\mathrm{GmMPK6}$ itself responsible} for the cell death on GmMPK6-overexpressing plants?

The enhanced expression of AtMPK6 in GmMPK6-overexpressing plants could be due to the secondary effect of cell death and activated defense responses caused by overexpression of GmMPK6. Activation of MPK6 activity but not just induction of the transcript itself alone leads to cell death and defense responses. Among 15 transgenic plants, only six of them showed cell death phenotypes, implying a possibility that the cell death on these six plants was triggered by activation of both GmMPK6 and endogenous AtMPK6 due to the possible

\section{Col-0}
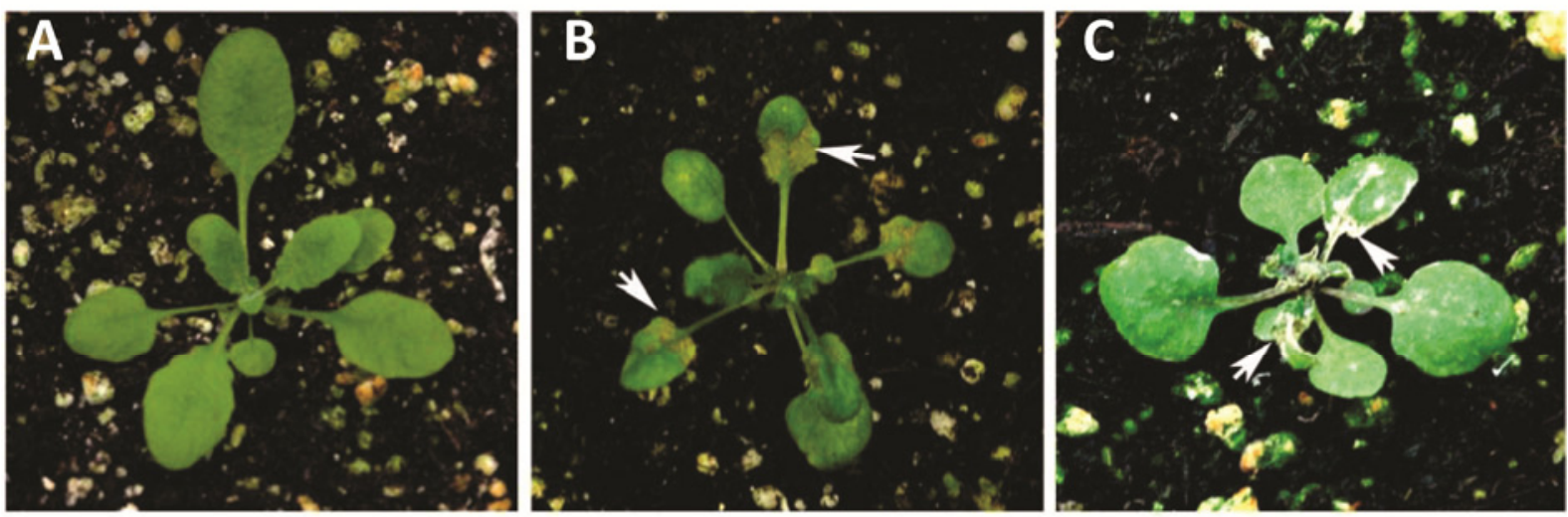

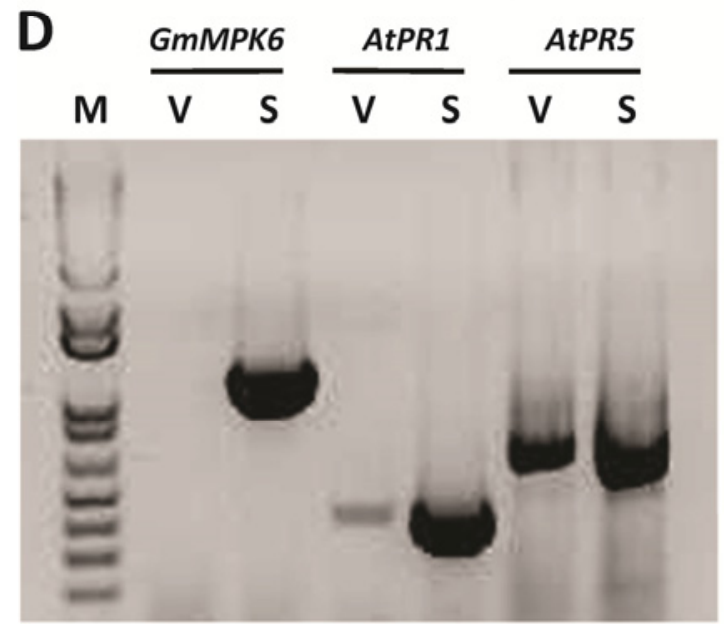

Fig. 6. Ectopic overexpression of GmMPK6 in Arabidopsis results in hypersensitive response (HR)-like cell death and pathogenesis-related (PR) gene induction. A, No HR-like cell death was observed on the leaves of Col-0 plants. B and $\mathbf{C}$, Examples of spontaneous HR-like cell death (see arrows) on the leaves of 6 of 15 transgenic Arabidopsis plants that ectopically overexpress GmMPK6. These plants eventually died before bolting. Note: Images in C and B were taken at different focal distance. D, Reverse-transcriptase polymerase chain reaction showed that the transgene (GmMPK6) was expressed at high level and that expression of AtPRI and AtPR5 mRNAs were constitutively activated in the transgenic Arabidopsis lines. V = vector control plants and S = GmMPK6silenced plants. 
presence of pathogens or abiotic stress in their micro-environment. Because the transcript level of MPK6 is much higher in these transgenic plants (both GmMPK6 and AtMPK6), activation of MPK6 activity in GmMPK6-overexpressing plants could be much stronger than in WT control plants. However, there could be a threshold for MPK6 transcript or MPK6 activity to trigger the cell death because transient overexpression of GmMPK6 in N. benthamiana leaves triggered cell death (Fig. 5) and a basal level of MPK6 activity is required for the growth and development in Arabidopsis (Müller et al. 2010; Wang et al. 2008). Therefore, the cell death and activated defense responses are regulated at both the transcriptional and post-translation level.

\section{MKK4-MPK6 is a common module}

in defense response in different species.

MAPKKK, MAPKK, and MPK form complicated signaling modules in plants. MEK2/MKK4/5-MPK6 is a conserved module involved in defense responses across plant species (Yang et al. 2001). However, other MKK also function upstream of MPK6 to form distinct modules involved in stomata patterning, ethylene production, and abiotic stress pathways (Bergmann et al. 2004; Wang et al. 2007; Yoo et al. 2008). The fact that overexpression of both GmMKK4 and GmMPK6 resulted in HR-like cell death in N. benthamiana or Arabidopsis (Fig. 5) and GmMKK4 interacted with GmMPK6 in the BiFC assay (Fig. 7) indicates that the GmMKK4-GmMPK6 module is also operating in soybean defense responses. A more rapid cell death triggered by $G m M K K 4^{D D}(2.5 \mathrm{dpi})$ than by GmMKK4 (3.5 dpi) (Fig. 5) and no cell death triggered by GmMKK4 ${ }^{\mathrm{KR}}$ (Fig. 5) further confirmed that phosphorylation of GmMKK4 is needed in order for it to induce cell death. The domains of MEK2 and MKK4 important for MKK4-MPK6 interactions have not yet been identified. It is not known whether the mutations at phosphorylation sites have any impact on the MKK4-MPK6 interaction. Our results clearly showed that mutations at phosphorylation sites of GmMKK4 did not interfere with its ability to interact with GmMPK6
(Fig. 7), indicating that GmMKK4 interacts with GmMPK6 independent of its phosphorylation status. The MAPKKK functioning upstream of GmMKK4-GmMPK6 remain to be identified in soybean.

\section{Conclusion.}

The positive roles of MPK6 and SIPK on cell death and disease resistance have been firmly established in model plants such as Arabidopsis, $N$. benthamiana, and tobacco (Colcombet and Hirt 2008; Pitzschke et al. 2009; Rasmussen et al. 2012). However, the negative roles of MPK6 on defense responses had not been reported until recently (Kobayashi et al. 2010). Our studies showed that knocking down GmMPK6 resulted in enhanced basal immunity against two unrelated pathogens in soybean. However, Rsvl-mediated ETI against SMV was not affected in GmMPK6-silenced plants (data not shown). Interestingly, silencing SIPK in tobacco enhances PTI against TMV but breaks $N$-mediated ETI (Kobayashi et al. 2010). These results add another layer of complexity to the MPK6-mediated defense signaling pathway and suggest that divergent roles of MPK6 exist in different plant species.

\section{MATERIALS AND METHODS}

\section{Plant materials.}

Seed of 'Williams 82' used in this study were harvested from greenhouse-grown plants previously indexed for the absence of BPMV and SMV (Zhang et al. 2009, 2010). Soybean plants were maintained in the greenhouse or growth chamber at $22^{\circ} \mathrm{C}$ with a photoperiod of $16 \mathrm{~h}$.

\section{BPMV-mediated VIGS.}

BPMV strains, BPMV VIGS constructs, and inoculation of soybean seedlings with DNA-based BPMV constructs via biolistic particle bombardments using a Biolistic PDS-1000/He system (Bio-Rad Laboratories, Hercules, CA, U.S.A.) have been previously described (Zhang et al. 2010; Zhang et al. 2009). The orthologs of Arabidopsis MPK or MKK in soybean

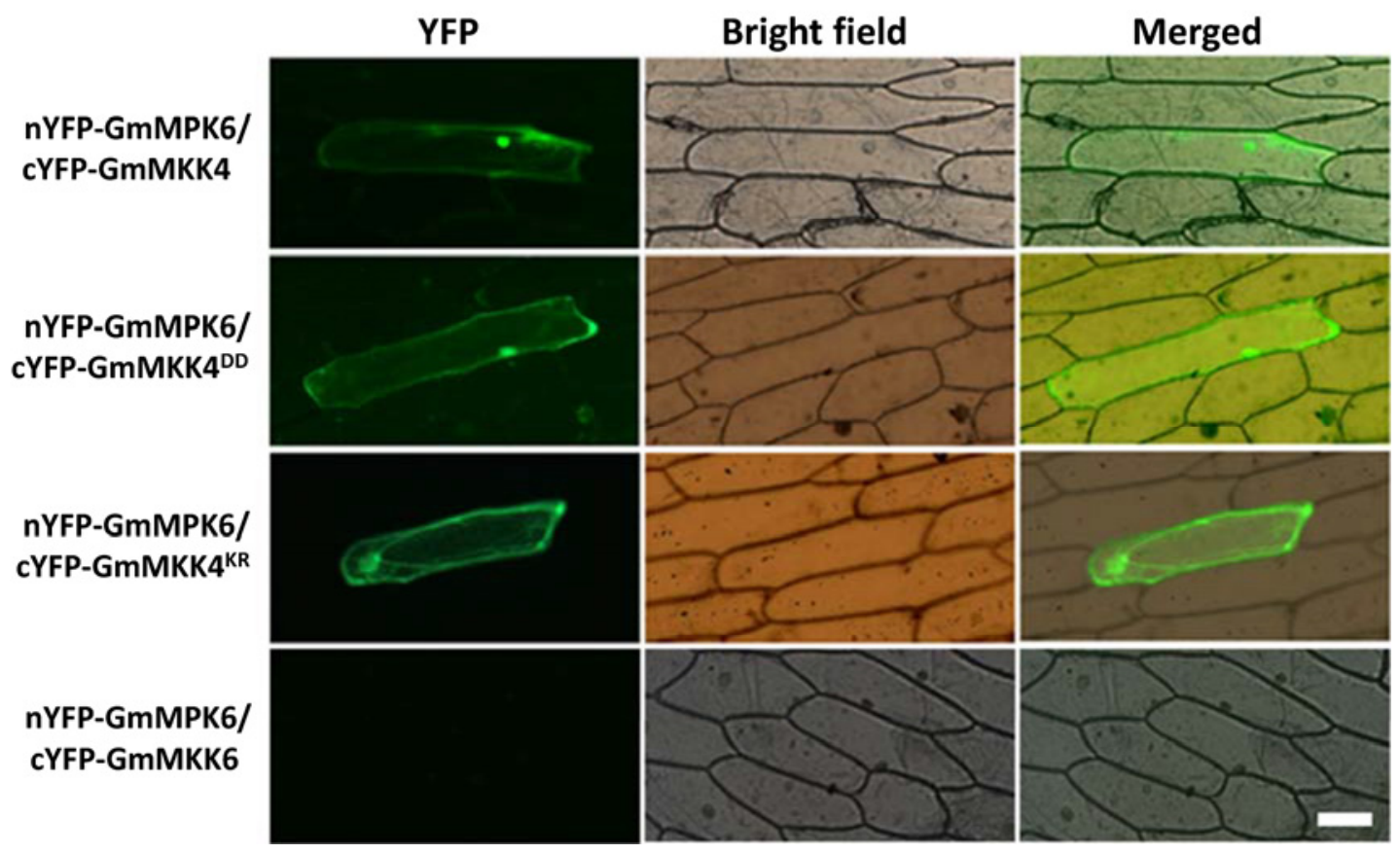

Fig. 7. GmMPK6 interacts with GmMKK4 in vivo and this interaction is independent of the phosphorylation status of GmMKK4. Yellow fluorescent protein (YFP) epifluorescence (left panels), bright field (central panel), and merged (right panels) images of onion epidermal cells co-bombarded with constructs expressing different fusion proteins as indicated. Co-expression of nYFP-GmMPK6 and cYFP-GmMKK6 was used as negative control. Bar $=100 \mu \mathrm{m}$. 
genome were identified by reciprocal BLASTn searches between Arabidopsis sequences and soybean gene models available in the Phytozome database. A fragment of GmMPK6 was amplified by PCR using the following primers: GmMPK6-F (Glyma02g15690): 5'-aagGGATCCCCAGAGCTTCTGTTGA

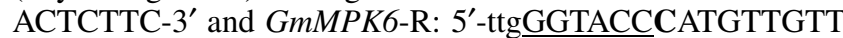
CAAAGTCAAAGTTGAAGG-3'. The underlined sequences are $B a m H I$ and $K p n I$ restriction sites, respectively, that were used to clone the PCR fragment into the BPMV VIGS vector. The bold letter indicates an extra nucleotide in reverse primers needed to maintain the reading frame.

\section{RNA isolation, RT-PCR, and qPCR.}

RNA isolation and RT-PCR were performed as described elsewhere (Liu et al. 2005). The qPCR tests were performed using an iCycler (Bio-Rad Laboratories) and the Platinum SYBRGreen qPCR SuperMix UDG (Invitrogen, Carlsbad, CA, U.S.A.). Total RNA samples were treated with DNaseI according to the manufacturer's instructions (Invitrogen). The primers used for qPCR were: GmMPK6-F: 5'-CAAACAGAG CAATGGAAGGAG-3'; GmMPK6-R: 5'-TATTCTCGATCCC CATAGCC-3'; Gm $\beta$-actin-F: 5'-GAGCTATGAATTGCCTGA TGG-3'; Gm $\beta$-actin-R: 5'-CGTTTCATGAATTCCAGTAGC-3'; GmPRl-F: 5'-AGAGGCAGAGGTGGGTTCT-3'; GmPRl-R: 5'-TCACCAACAAAGTTGCCAGG-3'; AtMPK6-F: ATGGAC GGTGGTTCAGGTC; and AtMPK6-R: CTATTGCTGATATT CTGGATTGAAAG.

\section{Western blot analysis.}

For GmMPK6 detection, total protein was extracted from BPMV-0 and BPMV-GmMPK6 or BPMV-GmMPK4 or BPMV-GmMKK1 plants and quantified using the Bradford method (Bio-Rad Laboratories). Equal amounts of total protein from different samples were loaded and were subjected to sodium dodecyl sulfate polyacrylamide gel electrophoresis. The protein was transferred to a polyvinylidene diflouride membrane using a Semi-Dry Trans blotting apparatus (BioRad Laboratories). The membrane was stained with Ponceau $\mathrm{S}$ before Western blot analysis. Western blot analysis was performed as described (Liu et al. 2005) using an antibody against AtMPK6 (Sigma (catalog number A7104) at a 1:2,000 dilution. The bands were visualized by incubating the membrane with nitroblue tetrazolium and 5-bromo-4-chloro-3-indolyl phosphate after secondary antibody incubation.

\section{SA quantification.}

SA was quantified using an Agilent 1100 high-performance liquid chromatograph with fluorometric detection (Agilent Technologies, Palo Alto, CA, U.S.A.) as described (Liu et al. 2011). The column was a 4.6-by-75-mm Agilent RR XDB $\mathrm{C} 18$, used with an isocratic mobile phase comprising $75 \% 20$ $\mathrm{mM}$ formate $(\mathrm{pH} 3.8), 20 \%$ methanol, and 5\% acetonitrile at a flow rate of $0.75 \mathrm{ml} / \mathrm{min}$ at $35^{\circ} \mathrm{C}$. SAG was measured after converting to free SA by acid hydrolysis. Recovery rates were determined using o-anisic acid as an internal standard and were typically greater than $60 \%$.

\section{SMV-N-GUS inoculation, GUS staining, and GUS foci measurements.}

SMV-N-GUS inoculation, GUS staining, and GUS foci measurements were described previously (Liu et al. 2011). Briefly, 18 dpi with BPMV empty vector (BPMV-0) or BPMV-GmMPK6 constructs by rub inoculation, the second fully expanded soybean trifoliolate leaves counting from the top were detached and biolistically inoculated with SMV-NGUS (Wang et al. 2006; Zhang et al. 2009). Following SMV$\mathrm{N}-\mathrm{GUS}$ inoculation, the detached leaves were placed on moist filter paper in petri dishes and incubated for 3 days on a lighted growth shelf until GUS staining (Jefferson et al. 1987). The leaves were imaged and photographed with a stereo microscope (Olympus SZH10) following GUS staining. The GUS foci were counted and their diameters measured using Soft Image System Analysis (IA Package, Olympus, Center Valley, PA, U.S.A.).

\section{Downy mildew infection.}

Downy mildew infection was previously described (Liu et al. 2011). The isolate of $P$. manschurica used in these studies was obtained from naturally infected plants in Ames, IA in 2008. The isolate is maintained by periodic transfer on soybean plants (Williams 82) in the greenhouse. Vector control and GmMPK6-silenced soybean plants were inoculated by spraying with a suspension of $P$. manschurica sporangia in deionized water $\left(10^{4}\right.$ sporangia/ml $)$. Plants were incubated in the dark at high humidity overnight and then moved to the greenhouse for 7 days. Symptoms were observed and samples for microscopy were collected 1 week after inoculation. Pathogen structures on and in plant tissues were visualized using a $\mathrm{KOH}-$ aniline blue staining procedure (Hood and Shew 1996). Tissues were excised from plants 1 week after inoculation. Tissue samples were placed in $1 \mathrm{M} \mathrm{KOH}$ for $24 \mathrm{~h}$, then heated in $1 \mathrm{M} \mathrm{KOH}$ for $30 \mathrm{~min}$ at $80^{\circ} \mathrm{C}$. Samples were rinsed in three changes of distilled water and soaked in $0.05 \%$ aniline blue in $0.7 \mathrm{M}$ $\mathrm{K}_{2} \mathrm{HPO}_{4}, \mathrm{pH} 9$, for $15 \mathrm{~min}$. Specimens were mounted in the same staining solution and observed with a Leitz Fluovert epifluorescence microscope with UV illumination (exciter filter BP 340-380; dichroic mirror RKP 400; barrier filter LP 430).

Autofluorescence was observed in leaf specimens that were fixed in boiling 95\% ethanol and cleared for several days in saturated chloral hydrate (Heath 1984). The cleared specimens were mounted in $50 \%$ glycerol and observed with blue illumination (exciter filter BP 420-490; dichroic mirror RKP 510; barrier filter LP 520).

\section{In vitro mutagenesis of $G m M K K 4$.}

In vitro mutagenesis was performed using the Gene Tailor Site-directed Mutagenesis System (Invitrogen). The primers used to generate $G m M K K 4^{D D}$ and $G m M K K 4^{K R}$ were GmMKK4-D1-F : 5'-TGGGTCGGATTCTGAATCAGGATA TGGATCCGTG-3'; GmKK4-D1/K-R: 5'-CTGATTCAGAATC CGACCCACCCCGAAGTC3'; GmMKK4-D2-F: 5'-AGACGA TGGATCCGTGCAATGATTCGGTGGGGAC-3'; GmMKK4D2/R-R: 5'-ATTGCACGGATCCATCGTCTGATTCAGAAT3'; GmMKK4-K-F: 5'-TGGGTCGGATTCTGAATCAGAAGA TGGATCCGTG-3'; and GmMKK4-R-F: 5'-AGACGATGGAT CCGTGCAATCGATCGGTGGGGAC-3'. The bold letters indicate the mutation sites.

First, the single amino acid mutants $214^{\mathrm{T} \rightarrow \mathrm{D}}$ and $214^{\mathrm{T} \rightarrow \mathrm{K}}$ were generated using $G m M K K 4$ as template and the $G m M K K 4-\mathrm{D} 1-\mathrm{F}$ or $G m M K K 4-\mathrm{K}-\mathrm{F}$ primers, respectively, with the $G m K K 4$ D1/K-R primer. The $G m M K K 4^{D D}$ and $G m M K K^{K R}$ mutants $\left(214^{\mathrm{T} \rightarrow \mathrm{D}} 220^{\mathrm{S} \rightarrow \mathrm{D}}\right.$ and $\left.214^{\mathrm{T} \rightarrow \mathrm{K}} 220^{\mathrm{S} \rightarrow \mathrm{R}}\right)$ were subsequently generated using the $214^{\mathrm{T} \rightarrow \mathrm{D}}$ and $214^{\mathrm{T} \rightarrow \mathrm{K}}$ single mutants as templates and the $G m M K K 4-\mathrm{D} 2-\mathrm{F}$ or $G m M K K 4-\mathrm{K}-\mathrm{F}$ primers, respectively, with the $G m M K K 4-\mathrm{D} 2 / \mathrm{R}-\mathrm{R}$ primer. The resulting GmMKK4 phosphorylation site mutants were confirmed by sequencing.

\section{BiFC analysis.}

The full-length cDNAs of GmMKK4 (Glyma07g00520), GmMPK6 (Glyma02g15690), and GmMPK4a (Glyma16g03670) were amplified from Williams 82 soybean plants, cloned into $\mathrm{pENTR/D}$ vector (Invitrogen), and then recombined via $\mathrm{LR}$ reaction (Invitrogen) into destination vectors $\mathrm{pE}-\mathrm{SPYCE}$ - 
GW and pE-SPYNE-GW that contain the C-terminal and Nterminal region of YFP, respectively (Walter et al. 2004). All constructs were confirmed by sequencing. The different combinations of constructs expressing SPYCE and SPYNE fusion proteins were co-bombarded into onion epidermal cells using Biolistic PDS-1000/He system (Bio-Rad Laboratories Laboratories) and images were captured with an inverted Axiophot microscope (Zeiss, Thornwood, NY, U.S.A.) equipped with a digital camera (Diagnostic Instruments, Sterling Heights, MI, U.S.A.).

The primers used for full-length cDNA amplification were GmMPK6-F: 5'-caccATGGAAGGAGGAGGAGCTG-3', GmMPK6-R: 5'-CTACTGCTGATACTCAGGGTTAAATG-3', GmMPK4-F: 5'-caccATGTCTGCTGTTGAGTCAGCTG-3', GmMK4-R: 5'-TCAGTAGACTGGTGGAACAGGATT-3', GmMKK4-F: $\quad 5^{\prime}$-caccATGAGGCCGATGCAACTG-3', and GmMKK4-R: 5'-CTAGGAAGGAAGAGGCCTTGG-3'. $G m M K K 4^{D D}$ and $G m M K K 4^{K R}$ were amplified using GmMKK4$\mathrm{F}$ and $G m M K K 4-\mathrm{R}$.

\section{ACKNOWLEDGMENTS}

We thank J. Dittman, J. Horner, and R. Den Adel for their excellent technical assistance; and A. Eggenberger for the 35-GUS construct. This work was supported by the NSF Plant Genome Research Program (award number 0820642), the Iowa Soybean Association, the United Soybean Board, the North Central Soybean Association, Hatch Act and State of Iowa Funds, the Natural Science Foundation of China (31371401), Qianjiang Talent Plan (2013R10074), and Natural Science Foundation of Zhejiang Province (award number: LY12C14001) to J.-Z. Liu.

\section{LITERATURE CITED}

Ahlfors, R., Macioszek, V., Rudd, J., Brosché, M., Schlichting, R., Scheel, D., and Kangasjärvi, J. 2004. Stress hormone-independent activation and nuclear translocation of mitogen-activated protein kinases in Arabidopsis thaliana during ozone exposure. Plant J. 40:512-522.

Asai, T., Tena, G., Plotnikova, J., Willmann, M. R., Chiu, W. L., GomezGomez, L., Boller, T., Ausubel, F. M., and Sheen, J. 2002. MAP kinase signaling cascade in Arabidopsis innate immunity. Nature 415:977-983.

Bergmann, D. C., Lukowitz, W., and Somerville, C. R. 2004. Stomatal development and pattern controlled by a MAPKK kinase. Science 304:1494-1497.

Bethke, G., Unthan, T., Uhrig, J. F., Pöschl, Y., Gust, A. A., Scheel, D., and Lee, J. 2009. Flg22 regulates the release of an ethylene response factor substrate from MAP kinase 6 in Arabidopsis thaliana via ethylene signaling. Proc. Natl. Acad. Sci. U.S.A. 106:8067-8072.

Bush, S. M., and Krysan, P. J. 2007. Mutational evidence that the Arabidopsis MAP kinase MPK6 is involved in anther, inflorescence, and embryo development. J. Exp. Bot. 58:2181-2191.

Chen, Z., and Klessig, D. F. 1991. Identification of a soluble salicylic acidbinding protein that may function in signal transduction in the plant disease-resistance response. Proc. Natl. Acad. Sci. U.S.A. 88:8179-8183.

Cho, S. K., Larue, C. T., Chevalier, D., Wang, H., Jinn, T. L., Zhang, S., and Walker, J. C. 2008. Regulation of floral organ abscission in Arabidopsis thaliana. Proc. Natl. Acad. Sci. U.S.A. 105:15629-15634.

Colcombet, J., and Hirt, H. 2008. Arabidopsis MAPKs: A complex signalling network involved in multiple biological processes. Biochem. J. 413:217-226.

Desikan, R., Hancock, J. T., Ichimura, K., Shinozaki, K., and Neill, S. J. 2001. Harpin induces activation of the Arabidopsis mitogen-activated protein kinases AtMPK4 and AtMPK6. Plant Physiol. 126:1579-1587.

Droillard, M. J., Boudsocq, M., Barbier-Brygoo, H., and Laurière, C. 2004. Involvement of MPK4 in osmotic stress response pathways in cell suspensions and plantlets of Arabidopsis thaliana: Activation by hypoosmolarity and negative role in hyperosmolarity tolerance. FEBS (Fed. Eur. Biochem. Soc.) Lett. 574:42-48.

Dutilleul, C., Benhassaine-Kesri, G., Demandre, C., Rézé, N., Launay, A., Pelletier, S., Renou, J. P., Zachowski, A., Baudouin, E., and Guillas, I. 2012. Phytosphingosine-phosphate is a signal for AtMPK6 activation and Arabidopsis response to chilling. New Phytol. 194:181-191.

Ellis, B. E. 2012. Postal code for a plant MAPK. Biochem. J. 446:e5-7. Published online.

Evrard, A., Kumar, M., Lecourieux, D., Lucks, J., von Koskull-Döring, P., and Hirt, H. 2013. Regulation of the heat stress response in Arabidopsis by MPK6-targeted phosphorylation of the heat stress factor HsfA2. PeerJ 1:e59. Published online.

Galletti, R., Ferrari, S., and De Lorenzo, G. 2011. Arabidopsis MPK3 and MPK6 play different roles in basal and oligogalacturonide- or flagellininduced resistance against Botrytis cinerea. Plant Physiol. 157:804-814.

Heath, M. C. 1984 Relationship between heat-induced fungal death and plant necrosis in compatible and incompatible interactions involving the bean and cowpea rust fungi. Phytopathology 74:1370-1376.

Hoang, M.H., Nguyen, X.C., Lee, K., Kwon, Y.S., Pham, H.T., Park, H.C., Yun, D.J., Lim, C.O., and Chung, W.S. 2012. Phosphorylation by AtMPK6 is required for the biological function of AtMYB41 in Arabidopsis. Biochem. Biophys. Res. Commun. 422:181-186.

Hood M.E., Shew H.D. 1996 Applications of KOH-aniline blue fluorescence in the study of plant-fungal interactions. Phytopathology 86:704708.

Hord, C.L., Sun, Y.J., Pillitteri, L.J., Torii, K.U., Wang, H., Zhang, S., and Ma, H. 2008. Regulation of Arabidopsis early anther development by the mitogen-activated protein kinases, MPK3 and MPK6, and the ERECTA and related receptor-like kinases. Mol. Plant 1:645-658.

Innes, R.W. 2001. Mapping out the roles of MAP kinases in plant defense. Trends Plant Sci. 6:392-394.

Jefferson, R.A., Kavanagh, T.A., and Bevan, M.W. 1987. GUS fusions: Beta-glucuronidase as a sensitive and versatile gene fusion marker in higher plants. EMBO J. 6:3901-3907.

Jin, H., Liu, Y., Yang, K.Y., Kim, C.Y., Baker, B., and Zhang, S. 2003. Function of a mitogen-activated protein kinase pathway in $N$ gene-mediated resistance in tobacco. Plant J. 33:719-731.

Joo, S., Liu, Y., Lueth, A., and Zhang, S. 2008. MAPK phosphorylationinduced stabilization of ACS6 protein is mediated by the non-catalytic C-terminal domain, which also contains the cis-determinant for rapid degradation by the $26 \mathrm{~S}$ proteasome pathway. Plant J. 54:129-140.

Khan, M., Rozhon, W., Bigeard, J., Pflieger, D., Husar, S., Pitzschke, A., Teige, M., Jonak, C., Hirt, H., and Poppenberger, B. 2013. Brassinosteroid-regulated GSK3/Shaggy-like kinases phosphorylate mitogen-activated protein (MAP) kinase kinases, which control stomata development in Arabidopsis thaliana. J. Biol. Chem. 288:7519-7527.

Kobayashi, M., Seo, S., Hirai, K., Yamamoto-Katou, A., Katou, S., Seto, H., Meshi, T., Mitsuhara, I., and Ohashi, Y. 2010. Silencing of WIPK and SIPK mitogen-activated protein kinases reduces Tobacco mosaic virus accumulation but permits systemic viral movement in tobacco possessing the $N$ resistance gene. Mol. Plant-Microbe Interact. 23:10321041.

Li, G., Meng, X., Wang, R., Mao, G., Han, L., Liu, Y., and Zhang, S. 2012a. Dual-level regulation of ACC synthase activity by MPK3/MPK6 cascade and its downstream WRKY transcription factor during ethylene induction in Arabidopsis. PLoS Genet. 8:e1002767. Published online.

Li, Z., Yue, H., and Xing, D. 2012b. MAP Kinase 6-mediated activation of vacuolar processing enzyme modulates heat shock-induced programmed cell death in Arabidopsis. New Phytol. 195:85-96.

Liu, J. Z., Blancaflor, E. B., and Nelson, R.S. 2005. The Tobacco mosaic virus 126-kilodalton protein, a constituent of the virus replication complex, alone or within the complex aligns with and traffics along microfilaments. Plant Physiol. 138:1853-1865.

Liu, J. Z., Horstman, H. D., Braun, E., Graham, M. A., Zhang, C., Navarre, D., Qiu, W. L., Lee, Y., Nettleton, D., Hill, J. H., and Whitham, S. A. 2011. Soybean homologs of MPK4 negatively regulate defense responses and positively regulate growth and development. Plant Physiol. 157:13631378.

Liu, Y., and Zhang, S. 2004. Phosphorylation of 1-aminocyclopropane-1carboxylic acid synthase by MPK6, a stress-responsive mitogen-activated protein kinase, induces ethylene biosynthesis in Arabidopsis. Plant Cell 16:3386-3399.

Mao, G., Meng, X., Liu, Y., Zheng, Z., Chen, Z., and Zhang, S. 2011. Phosphorylation of a WRKY transcription factor by two pathogenresponsive MAPKs drives phytoalexin biosynthesis in Arabidopsis. Plant Cell 23:1639-1653.

Meng, X., Wang, H., He, Y., Liu, Y., Walker, J. C., Torii, K. U., and Zhang, S. 2012. A MAPK cascade downstream of ERECTA receptor-like protein kinase regulates Arabidopsis inflorescence architecture by promoting localized cell proliferation. Plant Cell 24:4948-4960.

Meng, X., Xu, J., He, Y., Yang, K.Y., Mordorski, B., Liu, Y., and Zhang, S. 2013. Phosphorylation of an ERF transcription factor by Arabidopsis MPK3/MPK6 regulates plant defense gene induction and fungal resistance. Plant Cell 25:1126-1142.

Menke, F. L., van Pelt, J. A., Pieterse, C. M., and Klessig, D. F. 2004. Silencing of the mitogen-activated protein kinase MPK6 compromises disease resistance in Arabidopsis. Plant Cell 16:897-907.

Montillet, J. L., Leonhardt, N., Mondy, S., Tranchimand, S., Rumeau, D., Boudsocq, M., Garcia, A. V., Douki, T., Bigeard, J., Laurière, C., Chevalier, A., Castresana, C., and Hirt, H. 2013. An abscisic acid-inde- 
pendent oxylipin pathway controls stomatal closure and immune defense in Arabidopsis. PLoS Biol. 11:e1001513. Published online.

Mészáros, T., Helfer, A., Hatzimasoura, E., Magyar, Z., Serazetdinova, L., Rios, G., Bardóczy, V., Teige, M., Koncz, C., Peck, S., and Bögre, L. 2006. The Arabidopsis MAP kinase kinase MKK1 participates in defence responses to the bacterial elicitor flagellin. Plant J. 48:485-498.

Müller, J., Beck, M, Mettbach, U., Komis, G., Hause, G., Menzel, D., and Samaj, J. 2010. Arabidopsis MPK6 is involved in cell division plane control during early root development, and localizes to the pre-prophase band, phragmoplast, trans-Golgi network and plasma membrane. Plant J. 61:234-248.

Nguyen, X. C., Hoang, M. H., Kim, H. S., Lee, K., Liu, X. M., Kim, S. H., Bahk, S., Park, H. C., and Chung, W. S. 2012a. Phosphorylation of the transcriptional regulator MYB44 by mitogen activated protein kinase regulates Arabidopsis seed germination. Biochem. Biophys. Res. Commun. 423:703-708

Nguyen, X. C., Kim, S. H., Lee, K., Kim, K. E., Liu, X. M., Han, H. J., Hoang, M. H., Lee, S. W., Hong, J. C., Moon, Y. H., and Chung, W. S. 2012b. Identification of a C2H2-type zinc finger transcription factor (ZAT10) from Arabidopsis as a substrate of MAP kinase. Plant Cell Rep. 31:737-745.

Okawa, C., and Ishikawa, A. 2013. MPK6 contributes to non-host resistance to Magnaporthe oryzae in Arabidopsis thaliana. Biosci. Biotechnol. Biochem. 77:1320-1322.

Pitzschke, A., Schikora, A., and Hirt, H. 2009. MAPK cascade signalling networks in plant defence. Curr. Opin. Plant Biol. 12:421-426.

Rasmussen, M. W., Roux, M., Petersen, M., and Mundy, J. 2012. MAP kinase cascades in Arabidopsis innate immunity. Front. Plant Sci. 3:169.

Ren, D., Liu, Y., Yang, K.Y., Han, L., Mao, G., Glazebrook, J., and Zhang, S. 2008. A fungal-responsive MAPK cascade regulates phytoalexin biosynthesis in Arabidopsis. Proc. Natl. Acad. Sci. U.S.A. 105:56385643.

Shen, X., Yuan, B., Liu, H., Li, X., Xu, C., and Wang, S. 2010. Opposite functions of a rice mitogen-activated protein kinase during the process of resistance against Xanthomonas oryzae. Plant J. 64:86-99.

Takahashi, F., Yoshida, R., Ichimura, K., Mizoguchi, T., Seo, S., Yonezawa, M., Maruyama, K., Yamaguchi-Shinozaki, K., and Shinozaki, K. 2007. The mitogen-activated protein kinase cascade MKK3-MPK6 is an important part of the jasmonate signal transduction pathway in Arabidopsis. Plant Cell 19:805-818.

Teige, M., Scheikl, E., Eulgem, T., Dóczi, R., Ichimura, K., Shinozaki, K., Dangl, J. L., and Hirt, H. 2004. The MKK2 pathway mediates cold and salt stress signaling in Arabidopsis. Mol. Cell 15:141-152.

Walter, M., Chaban, C., Schütze, K., Batistic, O., Weckermann, K., Näke, C., Blazevic, D., Grefen, C., Schumacher, K., Oecking, C., Harter, K., and Kudla, J. 2004. Visualization of protein interactions in living plant cells using bimolecular fluorescence complementation. Plant J. 40:428438

Wan, J., Zhang, S., and Stacey, G. 2004. Activation of a mitogen-activated protein kinase pathway in Arabidopsis by chitin. Mol. Plant Pathol. 5:125-135.

Wang, H., Ngwenyama, N., Liu, Y., Walker, J. C., and Zhang, S. 2007. Stomatal development and patterning are regulated by environmentally responsive mitogen-activated protein kinases in Arabidopsis. Plant Cell 19:63-73.

Wang, H., Liu, Y., Bruffett, K., Lee, J., Hause, G., Walker, J. C., and Zhang, S. 2008. Haplo-insufficiency of MPK3 in MPK6 mutant background uncovers a novel function of these two MAPKs in Arabidopsis ovule development. Plant Cell 20:602-613.

Wang, L., Eggenberger, A., Hill, J., and Bogdanove, A. J. 2006. Pseudomonas syringae effector avrB confers soybean cultivar-specific avirulence on Soybean mosaic virus adapted for transgene expression but effector avrPto does not. Mol. Plant-Microbe Interact. 19:304-312.

Wang, P., Du, Y., Li, Y., Ren, D., and Song, C. P. 2010. Hydrogen peroxidemediated activation of MAP kinase 6 modulates nitric oxide biosynthesis and signal transduction in Arabidopsis. Plant Cell 22:2981-2998.

Xing, Y., Jia, W., and Zhang, J. 2009. AtMKK1 and AtMPK6 are involved in abscisic acid and sugar signaling in Arabidopsis seed germination. Plant Mol. Biol. 70:725-736.

Xu, J., and Chua, N. H. 2012. Dehydration stress activates Arabidopsis MPK6 to signal DCP1 phosphorylation. EMBO (Eur. Mol. Biol. Organ.) J. 31:1975-1984.

Xu, J., Li, Y., Wang, Y., Liu, H., Lei, L., Yang, H., Liu, G., and Ren, D. 2008. Activation of MAPK kinase 9 induces ethylene and camalexin biosynthesis and enhances sensitivity to salt stress in Arabidopsis. J. Biol. Chem. 283:26996-27006.

Yang, K. Y., Liu, Y., and Zhang, S. 2001. Activation of a mitogen-activated protein kinase pathway is involved in disease resistance in tobacco. Proc. Natl. Acad. Sci. U.S.A. 98:741-746.

Ye, Y., Li, Z., and Xing, D. 2013. Nitric oxide promotes MPK6-mediated caspase-3-like activation in cadmium-induced Arabidopsis thaliana programmed cell death. Plant Cell Environ. 36:1-15.

Yoo, S. D., Cho, Y. H., Tena, G., Xiong, Y., and Sheen, J. 2008. Dual control of nuclear EIN3 by bifurcate MAPK cascades in $\mathrm{C} 2 \mathrm{H} 4$ signalling. Nature 451:789-795.

Zhang, C., Yang, C., Whitham, S. A., and Hill, J. H. 2009. Development and use of an efficient DNA-based viral gene silencing vector for soybean. Mol. Plant-Microbe Interact. 22:123-131.

Zhang, C., Bradshaw, J., Whitham, S. A., and Hill, J. H. 2010. The development of an efficient multipurpose Bean pod mottle virus viral vector set for foreign gene expression and RNA silencing. Plant Physiol. 153:52-65.

Zhang, S., and Klessig, D. F. 1998. Resistance gene $N$-mediated de novo synthesis and activation of a tobacco mitogen-activated protein kinase by Tobacco mosaic virus infection. Proc. Natl. Acad. Sci. U.S.A. 95:7433-7438.

Zhang, S., and Liu, Y. 2001. Activation of salicylic acid-induced protein kinase, a mitogen-activated protein kinase, induces multiple defense responses in tobacco. Plant Cell 13:1877-1889.

Zhou, C., Cai, Z., Guo, Y., and Gan, S. 2009. An Arabidopsis mitogen-activated protein kinase cascade, MKK9-MPK6, plays a role in leaf senescence. Plant Physiol. 150:167-177.

\section{AUTHOR-RECOMMENDED INTERNET RESOURCE}

Phytozome database: www.phytozome.org 